\title{
Spin Drag in Noncondensed Bose Gases
}

\begin{abstract}
R. A. Duine and H. T. C. Stoof
Institute for Theoretical Physics, Utrecht University, Leuvenlaan 4, 3584 CE Utrecht, The Netherlands (Received 17 July 2009; revised manuscript received 1 September 2009; published 19 October 2009)

We show how time-dependent magnetic fields lead to spin motive forces and spin drag in a spinor Bose gas. We propose to observe these effects in a toroidal trap and analyze this particular proposal in some detail. In the linear-response regime we define a transport coefficient that is analogous to the usual drag resistivity in electron bilayer systems. Because of Bose enhancement of atom-atom scattering, this coefficient strongly increases as temperature is lowered. We also investigate the effects of heating.
\end{abstract}

DOI: 10.1103/PhysRevLett.103.170401

PACS numbers: 67.85. $-\mathrm{d}, 03.75 .-\mathrm{b}, 05.30 . \mathrm{Fk}$

Introduction: Coulomb drag and spin Coulomb drag.Understanding electronic transport [1] is one of the most important goals of condensed-matter physics. Indeed, materials are often characterized according to their transport properties. Furthermore, transport measurements provide important physical information. For example, the temperature dependence of transport coefficients, such as resistivity and conductivity, contain information on the elementary excitations and their scattering mechanisms. Moreover, the magnetic-field dependence allows for extracting the electronic phase-coherence length.

Analyzing results of transport measurements is complicated by the multitude of effects, such as electron-electron and electron-phonon interactions, that contribute. This problem is to a large extent circumvented in the Coulomb drag measurement of Gramila et al. [2], illustrated in Fig. 1, that aims at singling out the electronelectron interactions from the start. In this setup a bilayer of two-dimensional electron gases is separated by a tunnel barrier. A current $I$ is driven through the bottom layer that drags along the electrons in the other layer. In the top layer, an electrochemical potential is built up that cancels the drag and induces a voltage drop $V_{D}$, which results in a drag resistivity $\rho_{D}=V_{D} / I$. Originating from electron-electron interactions, this resistivity usually has the typical $\rho_{D} \propto T^{2}$ Fermi-liquid-like temperature dependence at low temperatures [3-5].

One approach in describing Coulomb drag is in terms of a function $\Gamma_{D}\left(v_{t}-v_{b}\right)$ that gives the rate of change of momentum per volume of the electron gases due to Coulomb scattering $[4,5]$ and that to a good approximation depends on the difference in the drift velocities $v_{t}$ and $v_{b}$ in the top and bottom layers. The equations of motion for these drift velocities are then given by

$$
\begin{gathered}
n_{t} m_{e} \frac{d v_{t}}{d t}=-\Gamma_{D}\left(v_{t}-v_{b}\right)-n_{t} e E_{t}-\frac{n_{t} m_{e} v_{t}}{\tau_{t}}, \\
n_{b} m_{e} \frac{d v_{b}}{d t}=+\Gamma_{D}\left(v_{t}-v_{b}\right)-n_{b} e E_{b}-\frac{n_{b} m_{e} v_{b}}{\tau_{b}} .
\end{gathered}
$$

Here $-e$ is the charge of the electron and $m_{e}$ its mass.
Furthermore, the electronic density and the electric field in the top (bottom) layer are denoted by $n_{t}\left(n_{b}\right)$ and $E_{t}\left(E_{b}\right)$, respectively. We have also added a scattering rate $1 / \tau_{t}$ and $1 / \tau_{b}$ for the top and bottom layer, respectively, that effectively takes into account intralayer Coulomb scattering, electron-phonon interactions, and disorder. In applying the above result to the situation in Fig. 1, we take $v_{t}=0$ since there is no current in the top layer. Solving for $v_{b}$ in the steady state, using that $\Gamma_{D} \ll n_{b} m_{e} \boldsymbol{v}_{b} / \tau_{b}$ because the interlayer scattering is much weaker than the intralayer scattering, we find that $v_{b}=-e E_{b} \tau_{b} / m_{e}$, as usual. In the linear-response regime the drift velocities are small, and we can use that $\Gamma_{D}(v) \simeq \Gamma_{D}^{\prime}(0) v$ incorporating the fact that there is no net momentum transfer if the drift velocities are equal. We then find that the electric field in the top layer is $E_{t}=-\Gamma_{D}^{\prime}(0) v_{b} / e n_{t}$. Using that the current density in the bottom layer $j_{b}=-n_{b} e v_{b}$, we have for the drag resistivity

$$
\rho_{D}=\frac{E_{t}}{j_{b}}=\frac{\Gamma_{D}^{\prime}(0)}{e^{2} n_{t} n_{b}} \text {. }
$$

This result shows that the drag resistivity is determined by the slope of the function $\Gamma_{D}(v)$ at $v=0$.

In an analogy to Coulomb drag, D'Amico and Vignale proposed spin Coulomb drag [6], which was observed by Weber et al. [7]. Spin drag, in which the layer degree of

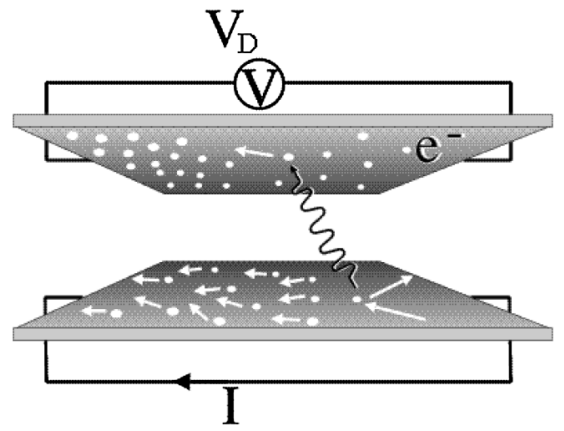

FIG. 1. Illustration of a Coulomb drag measurement: A pair of two-dimensional electron gases is separated by a tunnel barrier. A current is applied in one of the layers leading to a voltage drop in the other. (Adapted with permission from Ref. [19].) 
freedom from Coulomb drag is played by the spin of the electrons, is very similar to Coulomb drag. In this Letter, we study spin drag due to the short-range interatomic interactions in a spin-one Bose gas [8] in the normal state and propose an experiment to observe it making use of socalled spin motive forces. For this system, we derive equations of motion similar to Eqs. (1). The absence of disorder and an underlying lattice that supports phonons imply that the analogues of the scattering times $\tau_{b}$ and $\tau_{t}$ are infinite. Nonetheless, we recover a great deal of the phenomenology of conventional electronic transport. In particular, we define a transport coefficient analogous to $\rho_{D}$ which for bosons becomes large at small temperatures due to Bose enhancement, i.e., the enhanced scattering of bosons to states that are already occupied. In addition, we investigate heating effects and find that they are completely analogous to the usual joule heating in electronic systems.

Ultracold atomic gases and spin motive forces.-We consider ultracold atoms with hyperfine spin $F$ in a timeand position-dependent magnetic field with a direction given by the unit vector $\boldsymbol{\Omega}(\mathbf{x}, t)$, such that the Zeeman interaction reads $H_{Z}=-\Delta \boldsymbol{\Omega}(\mathbf{x}, t) \cdot \mathbf{F} / \hbar$, where $\mathbf{F}$ are the spin operators and $\Delta$ is an effective Zeeman splitting energy. If the magnetic-field direction is varying slowly in space and time, it is convenient to choose $\boldsymbol{\Omega}(\mathbf{x}, t)$ as the local spin quantization axis. In this frame of reference, spatial and temporal variation of the magnetic-field direction manifests itself as fictitious, or fixed-frame, electric and magnetic fields $\mathbf{E}$ and $\mathbf{B}$ [9] that are ultimately due to the spin Berry phase [10]. For atoms with spin projection $m_{F}$ these are given by

$$
\begin{aligned}
& E_{m_{F}, \alpha}=m_{F} \hbar \boldsymbol{\Omega} \cdot\left(\frac{\partial \boldsymbol{\Omega}}{\partial t} \times \nabla_{\alpha} \boldsymbol{\Omega}\right), \\
& B_{m_{F}, \alpha}=m_{F} \hbar \boldsymbol{\epsilon}_{\alpha \beta \gamma} \boldsymbol{\Omega} \cdot\left(\nabla_{\beta} \boldsymbol{\Omega} \times \nabla_{\gamma} \boldsymbol{\Omega}\right),
\end{aligned}
$$

where $\hbar$ is Planck's constant, $\epsilon_{\alpha \beta \gamma}$ is the three-dimensional fully antisymmetric Levi-Civita tensor, and a sum over repeated Cartesian indices $\alpha, \beta, \gamma \in\{x, y, z\}$ is implied. Note that, because the atoms are neutral, there are no real electromagnetic fields that couple to the atomic motion. In the context of ferromagnetic metals these fictitious electric and magnetic fields, respectively, underlie the phenomena of spin motive forces induced by moving domain walls and the topological Hall effect, both of which have been observed very recently $[11,12]$. In the context of cold atoms, the Aharonov-Bohm phase due to the fictitious magnetic field, in combination with phase coherence, has been used to imprint coreless vortices on $F=1$ spinor Bose-Einstein condensates $[13,14]$. For the existence of the fictitious electric and magnetic fields, phase coherence is, however, not required [9], and we can focus instead on the semiclassical regime using the equation of motion

$$
m \frac{d \mathbf{v}_{m_{F}}}{d t}=\mathbf{E}_{m_{F}}+\mathbf{v}_{m_{F}} \times \mathbf{B}_{m_{F}},
$$

for an atom with velocity $\mathbf{v}_{m_{F}}$ and spin projection $m_{F}$.
The specific geometry we consider is illustrated in Fig. 2 and consists of a toroidal trap with radius $R$ and effective cross section area $A$ in the transverse direction, created by a rapidly moving laser beam [15]. To implement the fictitious electric field we superpose a Ioffe-Pritchard magnetic trap. Fictitious electric fields along the torus are achieved by varying the axial bias field of the Ioffe-Pritchard trap, so that [14]

$$
\boldsymbol{\Omega}(\phi)=\Omega_{z}(t) \hat{z}+\sqrt{1-\Omega_{z}^{2}(t)}[\hat{r} \cos 2 \phi-\hat{\phi} \sin 2 \phi],
$$

in cylindrical coordinates $(r, \phi, z)$. Using Eq. (3), we then find that

$$
\mathbf{E}_{m_{F}}=2 \hbar m_{F} \frac{1}{R} \frac{d \Omega_{z}(t)}{d t} \hat{\phi},
$$

and $\mathbf{B}_{m_{F}}=0$. The adiabatic approximation that leads to the above holds when the time scale $T_{0}$ on which the direction of the external magnetic field is changed is much larger than the spin precession time $\hbar / \Delta$. Furthermore, this spin precession time should be smaller than the time it takes the atoms to encircle the torus. Since $\Delta$ is a large energy scale, these conditions are easily satisfied.

Spin drag.-We now specifically consider noncondensed bosonic atoms with $F=1$, e.g., sodium atoms. Furthermore, to study spin drag we consider the case that the trap is loaded with equal densities of atoms in spin state $|1\rangle$ with $m_{F}=+1$ and $|0\rangle$ with $m_{F}=0$. According to Eq. (6), the atoms in spin state $|1\rangle$ then feel a fictitious electric field $E_{1} \equiv E$ along the torus which accelerates them. The atoms in the $|0\rangle$ state feel no fictitious electric field but may accelerate due to spin drag, i.e., due to collisions with the other atoms.

To investigate the spin drag quantitatively, we use an effective one-dimensional Boltzmann equation for the distribution function $f_{1}(k, t)$ and $f_{0}(k, t)$ of the $m_{F}=1$ and $m_{F}=0$ atoms, with $\hbar k$ the momentum along the torus, given by

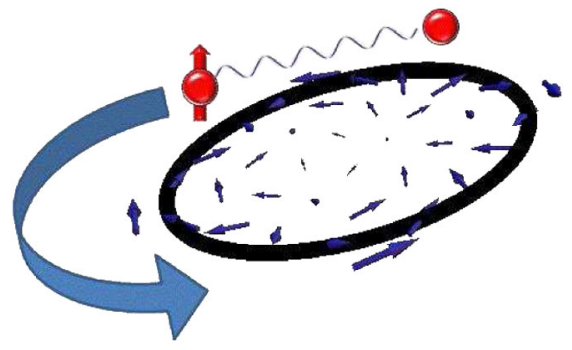

FIG. 2 (color online). Illustration of spin drag in a toroidal trap. The atoms with $m_{F}=1$ (red circle with arrow) are accelerated by the motive force due to the time-dependent quadrupole field (blue arrows) of the Ioffe-Pritchard trap. Because of interactions, the atoms with $m_{F}=0$ are dragged along. 


$$
\begin{aligned}
\frac{\partial f_{1}(k, t)}{\partial t}+\frac{E}{\hbar} \frac{\partial f_{1}}{\partial k}= & \Gamma_{\text {coll }}(k) \\
\equiv & \frac{2 \pi}{\hbar}\left(T_{01}^{2 B}\right)^{2} \int \frac{d k_{2}}{2 \pi} \int \frac{d k_{3}}{2 \pi} \int \frac{d k_{4}}{2 \pi}(2 \pi) \delta\left(k+k_{2}-k_{3}-k_{4}\right) \delta\left(\epsilon_{k}+\epsilon_{k_{2}}-\epsilon_{k_{3}}-\epsilon_{k_{4}}\right)\left\{\left[1+f_{1}(k, t)\right]\right. \\
& \left.\times\left[1+f_{0}\left(k_{2}, t\right)\right] f_{1}\left(k_{3}, t\right) f_{0}\left(k_{4}, t\right)-f_{1}(k, t) f_{0}\left(k_{2}, t\right)\left[1+f_{1}\left(k_{3}, t\right)\right]\left[1+f_{0}\left(k_{4}, t\right)\right]\right\},
\end{aligned}
$$

and where the equation of motion for $f_{0}(k, t)$ follows by taking $E=0$ and interchanging $f_{0}(k, t)$ and $f_{1}(k, t)$. The interspecies collisions are determined by the two-body $T$ matrix $T_{01}^{2 B}=4 \pi a \hbar^{2} / m A$, with $a$ the scattering length for collisions of atoms between atoms in states $|1\rangle$ and $|0\rangle$ and $m$ the atomic mass. The single-particle dispersion is $\epsilon_{k}=$ $\hbar^{2} k^{2} / 2 m$. On the right-hand side, we have ignored intraspin-species collisions which tend to restore local equilibrium and are zero in the approximations outlined below. Also note that, contrary to electronic transport in solidstate physics, there are no terms corresponding to elastic or electron-phonon collisions, because in cold-atom systems there is no disorder or an underlying ionic lattice.

Since the intraspecies collisions enforce local equilibrium for each spin species, we use a Bose-Einstein distribution function with nonzero drift velocity as an ansatz to solve the above equation. Specifically, we take $f_{1}(k, t)=N_{B}\left(\epsilon_{k-m v_{1}(t) / \hbar}\right)$ and $f_{0}(k, t)=N_{B}\left(\epsilon_{k-m v_{0}(t) / \hbar}\right)$, with $N_{B}(\epsilon)=\left[e^{\beta_{T}(\epsilon-\mu)}-1\right]^{-1}$ the Bose-Einstein distribution function at chemical potential $\mu$ and inverse thermal energy $\beta_{T}=1 / k_{B} T$. In the first instance we take the temperature constant in time. The time dependence of the chemical potential is determined by the conservation of the number of atoms in each spin state and is left implicit. From the Boltzmann equation we then find that [cf. Eqs. (1)]

$$
\begin{aligned}
& n m \frac{d v_{1}}{d t}=n E+\Gamma\left(v_{0}-v_{1}\right), \\
& n m \frac{d v_{0}}{d t}=-\Gamma\left(v_{0}-v_{1}\right),
\end{aligned}
$$

where $n$ is the one-dimensional density of each spin state, and the function that determines the rate of momentum transfer from species $|1\rangle$ to $|0\rangle$ is found from Eq. (7) as

$$
\Gamma\left(v_{0}-v_{1}\right)=-\int \frac{d k}{2 \pi} \Gamma_{\text {coll }}(k) \hbar k,
$$

with the right-hand side evaluated using the shifted BoseEinstein distribution functions. In Fig. 3, we plot this function for various values of the degeneracy parameter $n \Lambda$, with $\Lambda=\sqrt{2 \pi \hbar^{2} / m k_{B} T}$ the de Broglie wavelength. We find that in the classical limit $n \Lambda \rightarrow 0$ it is given by $\Gamma(v)=(4 \pi a \hbar n)^{2} \operatorname{Erf}(m \Lambda v / \hbar) / A^{2} m$. For increasing degeneracy $\Gamma(v)$ develops local maxima and minima at small $\left|v_{\max }\right|$ which are due to Bose enhancement of interspecies scattering.

From the equations of motion in Eqs. (8) we see that the sum of drift velocities increases indefinitely. The relative drift velocity $v=v_{1}-v_{0}$ can approach a steady state, provided the motive force $E$ is not too large. That is, from Eqs. (8) we find that if $n E \leq 2 \Gamma\left(v_{\max }\right)$ the system approaches a steady state where $d v / d t=0$. In the linearresponse regime $E$ and $v$ are small, and we have that $\Gamma\left(v_{0}-v_{1}\right) \simeq \Gamma^{\prime}(0)\left(v_{0}-v_{1}\right)$. Introducing the relativemomentum particle current $j=n\left(v_{1}-v_{0}\right)$, we have in linear response that $v_{1}-v_{0}=n E / 2 \Gamma^{\prime}(0)$. From this we define in the linear-response regime a resistivity $\rho \equiv$ $E / j=2 \Gamma^{\prime}(0) / n^{2}$ that is analogous to the drag resistivity in Eq. (2). For fermionic atoms this resistivity would vanish at small temperatures. For bosons it becomes larger due to Bose enhancement. This is further illustrated in the inset in Fig. 3, which shows $\Gamma^{\prime}(0)$ as a function of $1 /(n \Lambda)^{2} \propto T$. The low-temperature behavior of $\rho$ is found by using that at small temperatures $N_{B}(\epsilon) \rightarrow k_{B} T /(\epsilon-$ $\mu$ ), which, using Eqs. (7) and (9), yields $\rho \propto T^{-5 / 2}$. Note that the critical temperature for Bose-Einstein condensation is zero within our one-dimensional model.

The fact that the total kinetic energy of the system is increasing suggests that beyond-linear-response effects, such as heating, may be important. To investigate these, we have to solve Eqs. (8) coupled to an equation for the temperature. This equation is most easily derived by considering the total energy $U=\int\left(f_{1}+f_{0}\right) \epsilon_{k} d k / 2 \pi$. We evaluate this energy within our ansatz of Bose-Einstein distribution functions with nonzero drift velocities and time-dependent temperature $T(t)$ in this case. Using the Boltzmann equation in Eqs. (7) and (8), we find that

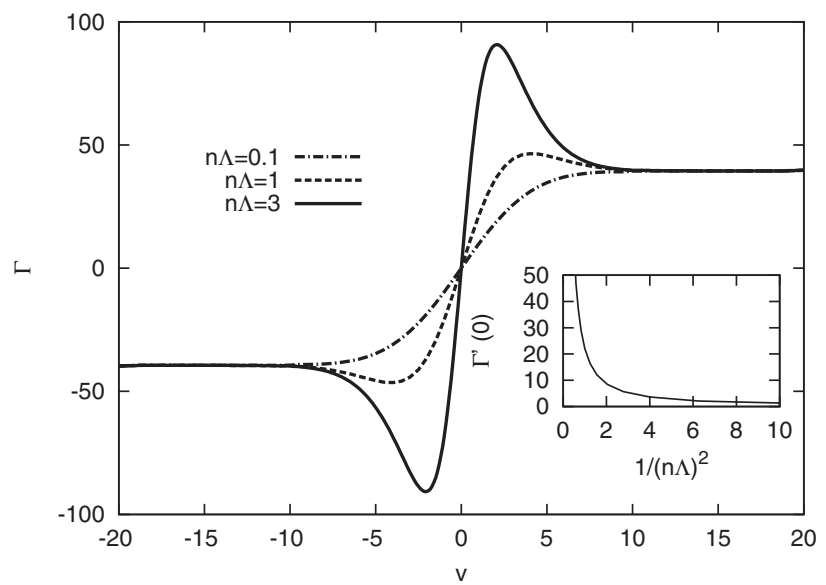

FIG. 3. Rate of momentum transfer $\Gamma$ in units of $A^{2} m /(2 a \hbar n)^{2}$. The difference of drift velocities is in units of $\hbar / m \Lambda$. The inset shows the derivative of $\Gamma$ at $v=0$. 


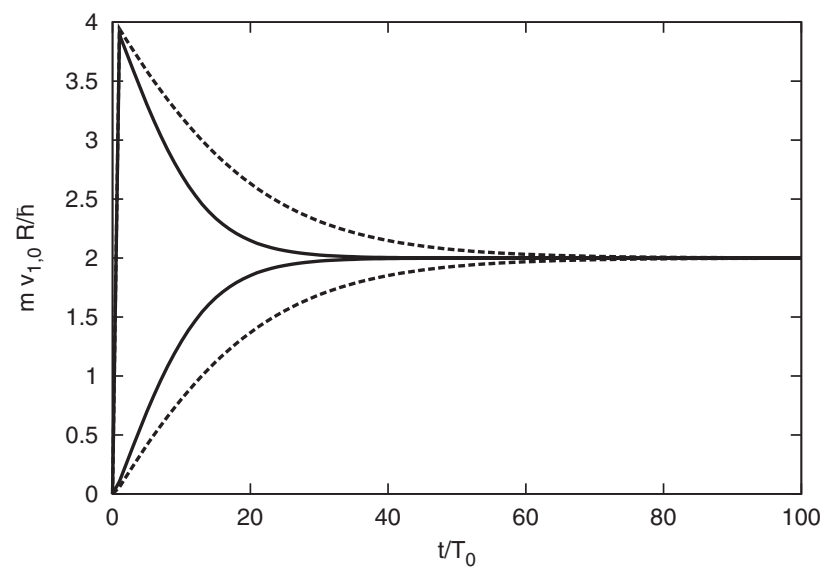

FIG. 4. Drift velocities of as a function of time, for $n \Lambda=45$ (solid lines) and $n \Lambda=30$ (dashed lines). The upper and lower curve correspond to $v_{1}$ and $v_{0}$, respectively.

$d Q / d t=n\left(v_{1}-v_{0}\right) \Gamma\left(v_{1}-v_{0}\right), \quad$ where $\quad Q \equiv U-$ $m n\left(v_{1}^{2}+v_{0}^{2}\right) / 2$. This energy is determined by the spread in velocities in the gas of atoms and is therefore a measure for its temperature. Evaluating the above using the linearresponse expression for the difference in drift velocities, we find that $d Q / d t=n \rho j^{2} / 2$, which is analogous to Ohmic heating in electronic systems.

We go beyond linear response by solving the equation for $d Q / d t$ coupled to Eqs. (8). We consider the case that the axial magnetic field of the Ioffe-Pritchard trap is inverted in a time $T_{0}$, so that $\Omega_{z}(t)=(2 t-1) / T_{0}$ for $0<$ $t<T_{0}$, and constant for $t>T_{0}$. This implies via Eq. (6) that $E=4 \hbar \hat{\phi} / R T_{0}$ for $0<t<T_{0}$ and zero for $t>T_{0}$. We consider specifically ${ }^{23} \mathrm{Na}$ atoms. As parameters we take $T_{0}=10 \mathrm{~ms}$ [14], $R=5 \mu \mathrm{m}$, and $T=400 \mathrm{nK}$. For the one-dimensional density we take $n=10^{12} \mathrm{~cm}^{-3} \times A$, with $A=\pi(5 \mu \mathrm{m})^{2}$ [16]. For these parameters $n \Lambda=45$. The result is shown in Fig. 4, together with the result for $n \Lambda=30$. We find that heating effects are negligible on the time scale that is shown. For each pair of curves the upper one corresponds to $v_{1}(t)$, which, due to acceleration by the motive force, acquires the value $v_{1} \simeq 4 \hbar / m R$ in the time $T_{0}$. The lower curve corresponds to $v_{0}$, which starts at $v_{0}(0)=0$. Because of the spin drag, the latter velocity also becomes nonzero, which can be experimentally measured by studying the momentum distribution after expansion. Note that the drag effect is larger for larger $n \Lambda$ due to the Bose enhancement.

Discussion and conclusions.-There are other experimental setups possible to observe spin-drag effects. For example, a cigar-shaped optical trap together with a magnetic-field gradient in the axial direction also leads to relative motion of the two spin species. We note that the hydrodynamic regime, where spin-drag effects should be large, has been realized recently in such cigar-shaped systems [17]. Another possibility is using a Raman tran- sition to convert a fraction of the atoms of one spin species to another and to set them into motion with a velocity determined by the recoil energy of the two-photon process. Such an experiment has already been performed with Bose-Einstein condensates [18]. However, to study spin drag, and other analogues of electronic transport, the noncondensed case is more suitable because the incoherent collisions of the thermal atoms, rather than the coherent interactions in a Bose-Einstein condensate, are analogous to the collisions of the electrons.

Yet another experimental possibility is to use a sinusoidally varying axial bias field of the Ioffe-Pritchard trap. This would lead to an ac electric field and the possibility to measure the frequency dependence of the transport coefficient $\rho$. Other interesting generalizations of the present work are including mesoscopic phase-coherence effects and effects of critical fluctuations. Drag effects can also be measured in Fermi gases [8], leading to another way to probe the many-body physics of these systems.

We thank Peter van der Straten, Robert Meppelink, and Johnny Vogels for pointing out to us various experimental possibilities to measure the spin drag and David Ritchie for allowing us to use Fig. 1. This work was supported by the Stichting voor Fundamenteel Onderzoek der Materie (FOM), the Netherlands Organization for Scientific Research (NWO), and the European Research Council (ERC) under the Seventh Framework Program (FP7).

[1] J. Rammer, Quantum Transport Theory (Westview Press, Boulder, CO, 1998), 1st ed.

[2] T. J. Gramila et al., Phys. Rev. Lett. 66, 1216 (1991).

[3] L. Zheng and A.H. MacDonald, Phys. Rev. B 48, 8203 (1993).

[4] A.-P. Jauho and H. Smith, Phys. Rev. B 47, 4420 (1993).

[5] A. G. Rojo, J. Phys. Condens. Matter 11, R31 (1999).

[6] I. D'Amico and G. Vignale, Phys. Rev. B 62, 4853 (2000).

[7] C. Weber et al., Nature (London) 437, 1330 (2005).

[8] For a discussion of spin-charge separation and drag in two-component Fermi gases, see M. Polini and G. Vignale, Phys. Rev. Lett. 98, 266403 (2007).

[9] A. Stern, Phys. Rev. Lett. 68, 1022 (1992).

[10] M. V. Berry, Proc. R. Soc. A 392, 45 (1984).

[11] S. A. Yang et al., Phys. Rev. Lett. 102, 067201 (2009).

[12] A. Neubauer et al., Phys. Rev. Lett. 102, 186602 (2009).

[13] T. Isoshima, M. Nakahara, T. Ohmi, and K. Machida, Phys. Rev. A 61, 063610 (2000).

[14] A. E. Leanhardt et al., Phys. Rev. Lett. 90, 140403 (2003).

[15] K. Henderson, C. Ryu, C. MacCormick, and M. G. Boshier, New J. Phys. 11, 043030 (2009).

[16] C. Ryu et al., Phys. Rev. Lett. 99, 260401 (2007).

[17] K. M. R. van der Stam, R. Meppelink, J. M. Vogels, and P. van der Straten, Phys. Rev. A 75, 031602(R) (2007).

[18] A. P. Chikkatur et al., Phys. Rev. Lett. 85, 483 (2000).

[19] http://www.sp.phy.cam.ac.uk/SPWeb/research/drag/ index.html. 